\title{
AN ULTRA-SMALL, LOW POWER ALL-OPTICAL FLIP-FLOP MEMORY ON A SILICON CHIP
}

Liu Liu $^{1 *}$, Rajesh Kumar ${ }^{1}$, Koen Huybrechts ${ }^{1}$, Thijs Spuesens ${ }^{1}$, Gunther Roelkens ${ }^{1}$, Erik-Jan Geluk ${ }^{2}$, Tjibbe de Vries ${ }^{2}$, Philippe Regreny ${ }^{3}$, Dries Van Thourhout ${ }^{1}$, Geert Morthier $^{1^{*}}$, Roel Baets ${ }^{1}$,

1. Photonics Research Group, INTEC Department, Ghent University-IMEC, B-9000 Gent, Belgium

2. COBRA Research Institute, Technische Universiteit Eindhoven, Postbus 513, 5600 MB Eindhoven, The Netherlands

3. Université de Lyon; Institut des Nanotechnologies de Lyon INL-UMR5270, CNRS, Ecole Centrale de Lyon, Ecully, F-69134, France

*liu.liu@intec.ugent.be; morthier@intec.ugent.be 
Future optical routing and switching will need high-speed and low-power optical processing of digital signals ${ }^{1}$. Ultra-small, low-power all-optical switching and memory elements, such as all-optical flip-flops (AOFFs), are often required ${ }^{1,2}$. For the fabrication of large-scale photonic integrated circuits, silicon-on-insulator (SOI) is considered ever more an attractive platform, owing to the compact devices it can offer and the complementary metal-oxide-semiconductor (CMOS) compatible fabrication process $^{3-5}$. Through heterogeneous integration of InP membranes onto $\mathrm{SOI}^{6-9}$, a single microdisk laser with diameter $7.5 \mu \mathrm{m}$ and coupled to an SOI wire waveguide is demonstrated here as an AOFF working in continuous-wave regime with electrical power consumption of only a few mWs, and allowing switching in 60 ps with just 1.8 fJ optical energy. The total power consumption and the device size are, to the best of our knowledge, the smallest reported so far at the telecom wavelength. This is also the only electrically-pumped AOFF on silicon, and built upon CMOS technology.

In optical packet switching networks, optical memory elements are required for holding the decisions from the optical header processors and for providing the control signals to the optical switches ${ }^{10}$. They are also necessary for buffering even the whole data packet in order to avoid the collision of data ${ }^{2}$. Such an optical memory can be realized through various delay lines, such as slow-light waveguides ${ }^{11,12}$, recirculating loops ${ }^{13}$, or micro-cavities ${ }^{14}$. However, these analog types of memories suffer from the small buffering time that can be achieved without significantly attenuating/distorting the signal. On the other hand, a digital optical memory, implemented via an AOFF, has the ability to store one bit of optical information in 
digital format with almost infinite duration, but the number of memory elements scales linearly with the number of bits that has to be stored. Obviously, the footprint and power consumption of single elements are of significant importance when they are going to be deployed in a larger scale memory.

Compact AOFFs have been demonstrated with a number of InP-based active devices, usually bistable laser diodes ${ }^{15-23}$. One of the smallest on-chip AOFFs reported at the telecom wavelength of $1.55 \mu \mathrm{m}$ consisted of two coupled ring lasers with each ring having a radius of $16 \mu \mathrm{m}$, and the flip-flop, completely fabricated in InP, having a total area of 40x18 $\mu \mathrm{m}^{215}$. However, the ring lasers each have a threshold current of $30 \mathrm{~mA}$ (and thus each have a power consumption of over $30 \mathrm{~mW}$ ) and can only operate in pulsed regime. Single InP ring lasers have been demonstrated as AOFF as well, but with diameters in excess of $50 \mu \mathrm{m}$, switching times over $100 \mathrm{ps}$, and total power consumption of several tens of $\mathrm{mW}^{16-21}$. Another small AOFF was based on the polarization switching in a vertical cavity surface emitting laser (VCSEL). While switching energies of $0.3 \mathrm{fJ}$ and switching times of 7 ps have been demonstrated at $980 \mathrm{~nm}$ using such an $\mathrm{AOFF}^{22}$, the switching energies increased to several $\mathrm{fJ}$ at a wavelength of $1.55 \mu \mathrm{m}^{23}$. The VCSEL at this wavelength required a bias current slightly above $14 \mathrm{~mA}$. Moreover, VCSELs are also less suited for on-chip integration with other devices.

On the other hand, to build such an electrically-pumped AOFF on SOI is not simple, since laser emission in silicon is still difficult due to its indirect bandgap. Although all-silicon 
lasers have been demonstrated using various approaches ${ }^{24,25}$, their performance is still far from sufficient for complex applications like AOFFs. Through optical pumping, though, optical bistability on silicon has been demonstrated based on the absorption induced thermo-optic or free carrier effect using small ring resonators or photonic crystal cavities ${ }^{26,27}$. These effects are typically slow in silicon and require a high input optical power. Another possibility is to employ the Kerr nonlinearity. For instance, fast, bistable switches have been proposed theoretically with photonic crystal cavities ${ }^{28}$. However, in practice the aforementioned slow effects always impair the instantaneous Kerr response in silicon. Although a silicon-organic hybrid waveguide has been introduced to avoid this ${ }^{29}$, no trials were reported to further develop it into AOFFs. Recently, III-V/SOI heterogeneous integration has been shown to give good lasing performance in silicon ${ }^{6-9}$. This technology is CMOS compatible, allows the combination of III-V materials and SOI circuits at their best quality, and can also provide dense, large-scale integration of devices. By using this approach, compact microdisk lasers have been fabricated with diameters as small as $7.5 \mu \mathrm{m}$, and continuous-wave lasing operation with electrical pumping has been demonstrated ${ }^{7}$.

In this paper, we present for the first time AOFFs built from such a single microdisk laser on SOI. The adhesive die-to-wafer bonding technology using divinylsiloxane-benzocyclobutene (DVS-BCB) polymer was employed to achieve the III-V/SOI heterogeneous integration ${ }^{6}$. Figure 1(a) shows schematic drawings of the entire circuit and the bonded microdisk laser. Flip-flop operation in the proposed device is based on switching between the clockwise (CW) and counter clockwise (CCW) whispering gallery modes (WGMs), where the two stable 
states can be labeled as CW dominant or CCW dominant, and they can be distinguished by the optical power towards either of the two directions of the SOI waveguide. As compared to the double ring configuration ${ }^{2}$, the additional difficulty of aligning the lasing wavelength of each ring is avoided in the proposed structure, which increases the yield and reduces the biasing complexity. The microdisk lasers have a diameter of $7.5 \mu \mathrm{m}$ with a threshold current of only $0.33 \mathrm{~mA}$, and bistable unidirectional operation occurs slightly above $1.7 \mathrm{~mA}$ under continuous wave. The light from the lasers is coupled to straight SOI wire waveguides (with dimensions of $500 \times 220 \mathrm{~nm}^{2}$ ) which are then coupled to single mode fibers at both ends using grating couplers ${ }^{30}$. Switching between $\mathrm{CW}$ and $\mathrm{CCW}$ modes is demonstrated using optical pulses of $1.8 \mathrm{fJ}$ and with a switching time of $60 \mathrm{ps}$.

From theoretical work and numerical simulations it is known that the existence of bistable unidirectional behavior requires a large enough photon density in the WGMs of the disk laser, as well as a sufficiently small coupling between the $\mathrm{CW}$ and the $\mathrm{CCW}$ modes ${ }^{16}$. In our microdisks, large internal power density results from the good mode confinement in the InP membrane due to the high index contrast, but also from the special heat-sinking that has been applied (see Methods). The coupling between CW and CCW modes depends on the surface roughness of the disk sidewalls, on the coupling between the disk and the straight SOI waveguides, on the reflection feedback from the grating couplers and the fiber facets, and on the reflection at the interface between disk and straight waveguide. The sidewall surface roughness can be minimized by using an optimized lithography and etching process. Another important parameter is the coupling ratio between the WGM and the SOI waveguide, which 
is related to the DVS-BCB bonding layer thickness (the distance between the top of the SOI waveguide and the bottom of the III-V layer), as well as the position of the SOI waveguide with respect to the edge of the microdisk. In order to keep the influence of the external reflection feedback small, a small coupling ratio, i.e. a thick DVS-BCB bonding layer, is desired. On the other hand, a too thick DVS-BCB layer will result in a weak lasing output power, and the switching power needed for the flip-flop operation will also increase. A cross-sectional picture of a fabricated microdisk laser is shown in Fig. 1(b). The thickness of the DVS-BCB layer is about $250 \mathrm{~nm}$. In this configuration, the coupling ratio is estimated to be about $1 \%$. The exact reflection from the grating couplers is not known, but numerical simulations predict a value of about $-22 \mathrm{~dB}$. The reflections at the interface between disk and straight waveguide are considered to be less important, also due to the thick DVS-BCB bonding layer and the low coupling between the disk and the SOI waveguide. The coupling between $\mathrm{CW}$ and $\mathrm{CCW}$ modes due to all the reflections and scattering discussed above lifts their degeneracy. The resulting frequency splitting of the laser peaks is typically in the range of hundred $\mathrm{MHz}$ to $\mathrm{GHz}$, and thus can be detected as a resonance in the radio frequency spectrum of the output light. Measurements allowed us to conclude that the total coupling between CW and CCW modes is about $1.8 \times 10^{9} \mathrm{~s}^{-1}$.

Figure 2(a) gives a typical light-current (L-I) curve of the microdisk laser, measured from both ends of the SOI waveguide, and thus representing the $\mathrm{CW}$ and $\mathrm{CCW}$ mode power, respectively. Threshold current is as low as $0.33 \mathrm{~mA}$. The maximum power in the SOI waveguide is $21 \mu \mathrm{W}$, limited by the thermal rollover around $3.8 \mathrm{~mA}$. Figure $2(\mathrm{~b})$ presents the 
lasing spectrum at $3.8 \mathrm{~mA}$ bias, showing a single mode operation with side mode suppression ratio higher than $40 \mathrm{~dB}$. Bistable, unidirectional operation starts at $1.7 \mathrm{~mA}$. We did not observe any switching of the lasing direction in the unidirectional regime when increasing the bias current, which does occur in the large ring lasers ${ }^{15}$. Theoretical calculations predicted that this switching behavior only happens when the laser mode hops to another azimuthal order, i.e., several free spectral ranges (FSRs) away, due to self heating ${ }^{19}$. Such a mode-hopping is unlikely to happen in the present structure, since the microdisk cavity is so small that the FSR is larger than $30 \mathrm{~nm}$ as depicted in Fig. 2(b). Periodic oscillations can also be found in the L-I curves, which are an evidence of the reflection feedback from the grating coupler or the fiber facet.

To demonstrate the switching operation of the proposed AOFF, the experiment as sketched in Fig. 3 was performed. Assume that the microdisk laser works initially in the $\mathrm{CW}$ dominant state (Fig. 3(a)), where the CCW mode is suppressed and the power measured from the left side of the SOI waveguide is high. At a certain clock period, an optical reset pulse, at the same wavelength of the microdisk laser, is injected from the left side (Fig. 3(b)). This pulse will couple into the microdisk cavity. If the injected pulse is strong enough, it will invoke the CCW mode. Even after the pulse has passed through, the microdisk laser will stay in the CCW dominant state (Fig. 3(c)). In this case, the power at the left side of the SOI waveguide becomes low. Similarly, the $\mathrm{CW}$ dominant state can be recovered by injecting an optical set pulse from the right side (Fig. 3(d) and 3(e)). Note that this operation is actually equivalent to a conventional set/reset flip-flop in an electronic circuit. The microdisk laser was biased at 
$3.5 \mathrm{~mA}$, about twice the threshold current for the unidirectional operation $(1.7 \mathrm{~mA})$, since self switching due to noise can be observed if working too close to $\mathrm{it}^{21}$. If a continuous-wave light—or a sufficiently long pulse-is injected, reliable switching can be achieved with a power of $360 \mathrm{nW}$, which is about $-17 \mathrm{~dB}$ of the output power from the microdisk laser. As shown in Fig. 3(f) and 3(g), power extinction ratios of about $13 \mathrm{~dB}$ were measured at both sides of the SOI waveguide.

In order to test the switching speed and switching energy, a $10 \mathrm{GHz}$ lithium niobate modulator was used to generate the set/reset pulses, and a communication signal analyzer was used to record the output laser signal. Figure 4(a) shows the waveform of the injected pulse which has a width of 100 ps. As shown in Fig. 4(b), switching between the CW and the CCW modes was achieved with peak power as low as $18 \mu \mathrm{W}$ measured in the SOI waveguide, corresponding to a pulse energy of $1.8 \mathrm{fJ}$. An extinction ratio of $11 \mathrm{~dB}$ was obtained, slightly less than that in the static conditions, probably due to the spontaneous emission noise from the Er-doped fiber amplifier used to amplify the signal. Further increasing the peak power of injected pulses induced a strong relaxation oscillation at the switch-on transient, due to the deep depletion of the carriers. The injected set/reset pulses are also visible in Fig. 4(b). They cover the transient of the microdisk output, which makes it difficult to measure the exact switching time of the flip-flop. The residual reset pulses mainly come from the interface reflection at the cleaved facet of the access fiber. They can be suppressed, to some extent, by using index matching fluid between the access fibers and the SOI chip. The measured switch-off transient in this case is presented in Fig. 4(c). A switching time of 60 ps was 
obtained. We did not observe significant improvement of the switch-off time when increasing the pulse peak power, up to the point where the reflected reset pulse started to appear again in the waveform. On the other hand, the set pulses cannot be removed in the current design, since they are at the same wavelength of the microdisk output, and propagating in the same direction as well. Nevertheless, we can still conclude, from Fig. 4(d), that the switch-on time should be less than 100 ps. In principle, the switching time in such a small microdisk laser should be of the order of $\mathrm{ps,}$ as suggested by numerical simulations ${ }^{15,17}$. To measure such a fast transition, a short and strong pulse is required ${ }^{15,18}$, and a smart design to efficiently separate the microdisk laser output from the injected pulses has to be introduced as well. One solution is to add another SOI waveguide coupled to the microdisk, so that the input and output light signals can be isolated physically in different waveguides.

Table 1 summarizes several performance metrics for the existing electrically-pumped AOFFs working at communication wavelengths, including coupled ring lasers ${ }^{2}$, single ring lasers ${ }^{18,20}$, polarization bistable VCSELs ${ }^{23}$, and the microdisk lasers (this work). Obviously, the active device area and the electrical power consumption of the proposed microdisk laser AOFF are the smallest among the AOFFs. The switching energy is similar to that of the VCSEL-based AOFF at its lowest speed, and is still much better at a high speed. It is also less than the switching energy in the other two approaches. The measured switching speed is still outperformed by that of the coupled ring lasers, but with the potential to go faster as discussed above. The proposed device is also the only electrically-pumped AOFF built on 
SOI so far. This advantage makes it extremely versatile when integrating with other passive devices to perform dense on-chip data processing.

\section{Methods}

Device fabrication. The SOI circuit was fabricated with $193 \mathrm{~nm}$ deep ultraviolet lithography through ePIXfab silicon photonics platform (www.epixfab.eu). An unpatterned III-V die, grown by solid source molecular beam epitaxy, was bonded on top of the finished SOI wafer/chip by using DVS-BCB ${ }^{6}$. The III-V layer has a total thickness of $583 \mathrm{~nm}$, including three compressively strained InAsP quantum wells for providing transverse electric mode gain and a tunnel junction for a low loss p-contact. After removing the InP substrate, contact lithography was used to define the microdisk pattern to make sure the underlying SOI waveguide is well aligned to the edge of the disk, so that the WGM can efficiently couple out to the waveguide. The III-V layer was etched by inductively coupled plasma - reactive ion etching until a thin $\mathrm{n}$-doped $\mathrm{InP}$ lateral contact layer $(90 \mathrm{~nm})$ was reached. This $\mathrm{InP}$ contact layer was then removed where it is not necessary, and a Ti/Pt/Au metal layer was deposited on top of it to form the bottom contact. Then, the whole structure was covered by DVS-BCB. A via was opened through the DVS-BCB layer on the center of the microdisk, and another $\mathrm{Ti} / \mathrm{Pt} / \mathrm{Au}$ metal layer was deposited for the top contact. The Au layer, which also served as a heat sink, was made thick here $(600 \mathrm{~nm})$ in order to improve the heat dissipation under continuous-wave bias. Since the WGM is confined to the edge of the disk, this top metal layer does not result in substantial optical absorption losses. The DVS-BCB on part of the bottom contact metal was also etched away, so that it can be reached electrically. 
References:

1. Tucker, R. S. Role of Optics and Electronics in High-Capacity Routers. IEEE J.

Lightwave Technol. 24, 4655-4673 (2006).

2. Dorren, H. J., Calabretta, N. \& Raz, O. Scaling all-optical packet routers: how much buffering is required? [Invited]. J. Opt. Netw. 7, 936-946 (2008).

3. Bogaerts, W. et al. Nanophotonic waveguides in silicon-on-insulator fabricated with CMOS Technology. IEEE J. Lightwave Technol. 23, 401-412 (2005).

4. Tsuchizawa, T. et al. Microphotonics devices based on silicon microfabrication technology. IEEE J. Sel. Top. Quantum Electron. 11, 232-239 (2005).

5. Jalali, B. \& Fathpour, S. Silicon Photonics. IEEE J. Lightwave Technol. 24, 4600-4615 (2006).

6. Roelkens, G., Van Thourhout, D., Baets, R., Notzel, R. \& Smit, M. Laser emission and photodetection in an $\mathrm{InP} / \mathrm{InGaAsP}$ layer integrated on and coupled to a silicon-on-insulator waveguide circuit. Opt. Express 14, 8154-8159 (2006).

7. Van Campenhout, J. et al. Electrically pumped InP-based microdisk lasers integrated with a nanophotonic silicon-on-insulator waveguide circuit. Opt. Express 15, 6744-6749 (2007)

8. Fang, A. W. et al. Electrically pumped hybrid AlGaInAs-silicon evanescent laser. Opt. Express 14, 9203-9210 (2006).

9. Sun, X. et al. Electrically pumped hybrid evanescent Si/InGaAsP lasers. Opt. Lett. 34, 1345-1347 (2009). 
Dorren, H. J. S. et al. Optical packet switching and buffering by using all-optical signal processing methods. IEEE J. Lightwave Technol. 21, 2-12 (2003).

11. Vlasov, Y. A., O’Boyle, M., Hamann, H. F. \& McNab, S. J. Active control of slow light on a chip with photonic crystal waveguides. Nature 438, 65-69 (2005).

12. Xia, F. N., Sekaric, L. \& Vlasov, Y. Ultracompact optical buffers on a silicon chip. Nature Photon. 1, 65-71, (2007).

13. Park, H., Mack, J. P., Blumenthal, D. J. \& Bowers, J. E. An integrated recirculating optical buffer. Opt. Express 16, 11124-11131 (2008).

14. Tanaka, Y. et al. Dynamic control of the Q factor in a photonic crystal nanocavity. Nature Materials 6, 862-865 (2007).

15. Hill, M. T. et al. A fast low-power optical memory based on coupled micro-ring lasers. Nature 432, 206-209 (2004).

16. Sorel, M. et al. Operating Regimes of GaAs-AlGaAs Semiconductor Ring Lasers: Experiment and Model. IEEE J. Quantum Electron. 39, 1187-1195 (2003).

17. Yuan, G., Wang, Z. \& Yu, S. Dynamic Switching Response of Semiconductor Ring Lasers to NRZ and RZ Injection Signals. IEEE Photon. Technol. Lett. 20, 785-787 (2008).

18. Trita, A. et al. Dynamic Operation of All-Optical Flip-Flop based on a Monolithic Semiconductor Ring Laser. European Conference on Optical Communication, paper We2C3 (2008). 
19. Fürst, S., Pérez-Serrano, A., Scirè, A., Sorel, M. \& Balle, S. Modal structure, directional and wavelength jumps of integrated semiconductor ring lasers: Experiment and theory. Appl. Phys. Lett. 93, 251109 (2008).

20. Mezosi, G., Strain, M. J., Furst, S., Wang, Z. \& Sorel, M. Unidirectional bistability in AlGaInAs Microring and Microdisk Semiconductor Lasers. IEEE Photon. Technol. Lett. 21, 88-90 (2009).

21. Gelens, L. et al. Exploring Multistability in Semiconductor Ring Lasers: Theory and Experiment. Phys. Rev. Lett. 102, 193904 (2009).

22. Mori, T., Yamayoshi, Y. \& Kawaguchi, H. Low-switching energy and high-repetition-frequency all-optical flip-flop operations of a bistable vertical-cavity surface-emitting laser. Appl. Phys. Lett. 88, 101102 (2006).

23. Katayama, T., Kitazawa, T. \& Kawaguchi, H. All-Optical Flip-Flop Operation using 1.55 $\mu \mathrm{m}$ Polarization Bistable VCSELs. Conference on Lasers and Electro-Optics/Quantum Electronics and Laser Science Conference, paper CME5 (2008).

24. Pavesi, L., Dal Negro, L., Mazzoleni, C., Franzò, G. \& Priolo, F. Optical gain in silicon nanocrystals. Nature 408, 440-444 (2000).

25. Rong, H. et al. A continuous-wave Raman silicon laser. Nature 433, 725-728 (2005).

26. Almeida, V. R. \& Lipson, M. Optical bistability on a silicon chip. Opt. Lett. 29, 2387-2389 (2004).

27. Tanabe, T., Notomi, M., Mitsugi, S., Shinya, A. \& Kuramochi, E. Fast bistable all-optical switch and memory on a silicon photonic crystal on-chip. Opt. Lett. 30, 2575-2577 (2005). 
28. Yanik, M. F., Fan, S. \& Soljacic, M. High-contrast all-optical bistable switching in photonic crystal. Appl. Phys. Lett. 83, 2739-2741 (2003).

29. Koos, C. et al. All-optical high-speed signal processing with silicon-organic hybrid slot waveguides. Nature Photon. 3, 216-219 (2009).

30. Taillaert, D. et al. Grating couplers for coupling between optical fibers and nanophotonic waveguides. Jpn. J. Appl. Phys. 45, 6071-6077 (2006). 
Acknowledgements:

This work is supported by the European FP7 ICT-projects HISTORIC, WADIMOS, and PhotonFAB, the Belgian Fund for Scientific Research Flanders (FWO), and the IAP-project "Photonics@be". The work of K. Huybrechts and T. Spuesens is supported by the Institute for the Promotion of Innovation through Science and Technology (IWT) under a specialization grant. We thank Marie Verbist for taking the cross-section picture. We acknowledge the assistance from Steven Verstuyft during the device fabrication. 
Author contributions:

D.V.T., G.M., and R.B. coordinated and supervised the project. L.L., R.K., T.S., G.R., E.G.,

T.d.V., and P.R. fabricated the devices. L.L., R.K., and K.H. performed the measurement.

L.L. and G.M. wrote the manuscript. 
Competing interests statement:

The authors declare that they have no competing financial interests. 
Figure legends:

Figure 1 | Structure of the microdisk laser based AOFF integrated on an SOI waveguide circuit. a, Schematic structures of the whole circuit (the grating coupler for fiber access is depicted only on one end of the SOI waveguide) and the microdisk laser (inset). b, Scanning electron microscope picture of the cross-section (made by focused ion beam etching) of a fabricated device, showing the disk cavity, the metal contacts, and the SOI waveguide.

Figure 2 | L-I curves and spectrum of a $7.5 \mu \mathrm{m}$ diameter microdisk laser. a, L-I curves for the two competing modes (CW and $\mathrm{CCW}$ ) of the microdisk laser under continuous-wave operation and at room temperature. $\mathbf{b}$, Lasing spectrum for the $\mathrm{CW}$ mode at a bias of $3.8 \mathrm{~mA}$. All powers are calculated inside the SOI waveguide by taking into account the coupling efficiency of the grating coupler.

\section{Figure 3 | Diagrams of the flip-flop experiment and measured power in the SOI}

waveguide at a low switching speed. a, The microdisk laser (wavelength at $1572.198 \mathrm{~nm}$ )

initially works in the CW dominant state. b, An external optical pulse (wavelength at 1572.2 $\mathrm{nm}$ ) is injected from the left side, and $\mathbf{c}$, switches the microdisk laser to the $\mathrm{CCW}$ dominant state when it passes through. $\mathbf{d} \& \mathbf{e}$, Similar to b \& c but with injection from the right side and the laser switches back to the CW dominant state. $\mathbf{f} \boldsymbol{\&} \mathbf{g}$, Power measured at the left and right ends of the SOI waveguide, respectively. The vertical dashed lines indicate approximately the moments at which diagrams a-e happened. 
Figure 4 | High-speed measurement of the switching characteristics. a, Waveform of the injected optical pulse (central wavelength at $1572.23 \mathrm{~nm}$ ). $\mathbf{b}$, Waveform of the measured optical signal at one side of the SOI waveguide. $\mathbf{c} \& \mathbf{d}$, Details of the switch-on and switch-off transients, respectively, after applying the index matching fluid to suppress the appearance of the reset pulses. 
Tables:

Table 1 | Comparison of several performance metrics for AOFFs.

\begin{tabular}{|c|c|c|c|c|c|}
\hline & Device size & $\begin{array}{c}\text { Electrical } \\
\text { bias current }\end{array}$ & Switching energy & $\begin{array}{c}\text { Switching } \\
\text { speed }\end{array}$ & $\begin{array}{c}\text { Material } \\
\text { system }\end{array}$ \\
\hline $\begin{array}{c}\text { Coupled ring } \\
\text { laser }\end{array}$ & $2 \times 16 \times 16 \mu \mathrm{m}^{2}$ & $>2 \times 30 \mathrm{~mA}$ & $5.5 \mathrm{fJ}$ & $20 \mathrm{ps}$ & $\mathrm{InP}$ \\
\hline $\begin{array}{c}\text { Single ring } \\
\text { laser }\end{array}$ & $>50 \times 50 \mu \mathrm{m}^{2}$ & $>60 \mathrm{~mA}$ & $4 \mathrm{pJ}$ & $130 \mathrm{ps}$ & $\mathrm{InP}$ \\
\hline VCSEL $^{23}$ & $10 \times 10 \mu \mathrm{m}^{2}$ & $14.5 \mathrm{~mA}$ & $\begin{array}{c}1.8 \mathrm{fJ}(\text { at } 50 \mathrm{MHz})^{*} \\
10 \mathrm{fJ}(\text { at } 3.1 \mathrm{GHz})\end{array}$ & $300 \mathrm{ps}^{\dagger}$ & $\mathrm{InP}$ \\
\hline $\begin{array}{c}\text { Microdisk laser } \\
\text { (this work) }\end{array}$ & $7.5 \times 7.5 \mu \mathrm{m}^{2}$ & $3.5 \mathrm{~mA}$ & $1.8 \mathrm{fJ}$ & $60 \mathrm{ps}$ & $\begin{array}{c}\mathrm{InP} \text { on } \\
\mathrm{SOI}\end{array}$ \\
\hline
\end{tabular}

\footnotetext{
* Taken as an average of the set and rest pulse energy.

$\dagger$ Taken as an inverse of the highest operation frequency, i.e., 3.1 GHz.
} 


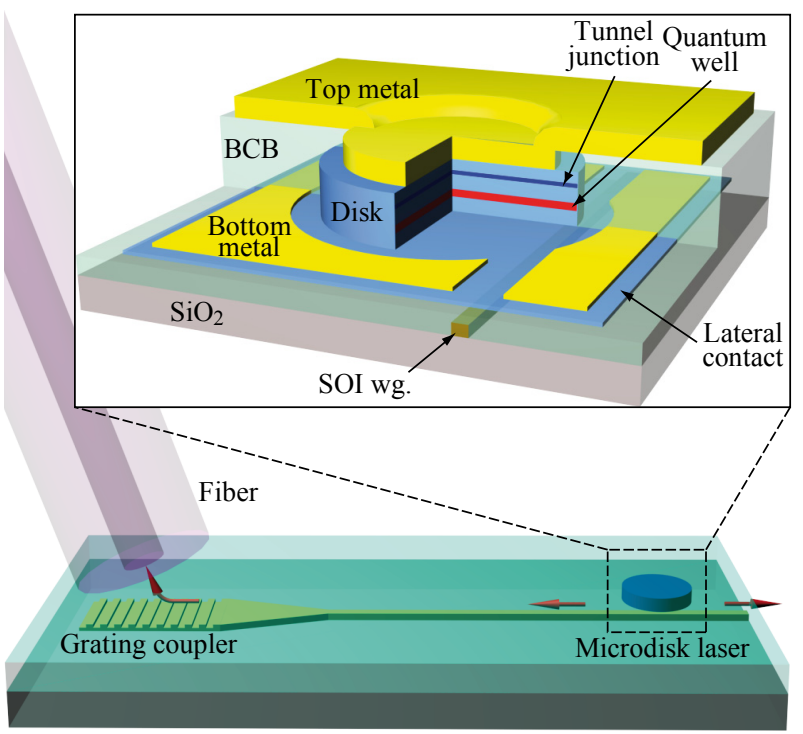

(a)

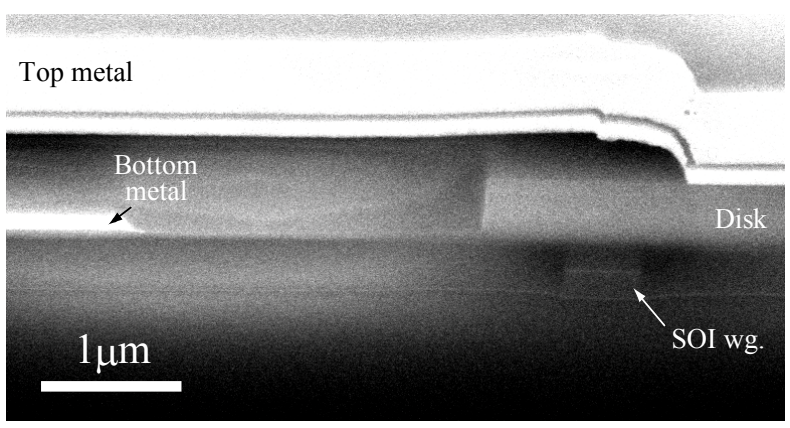

(b)

Figure 1 

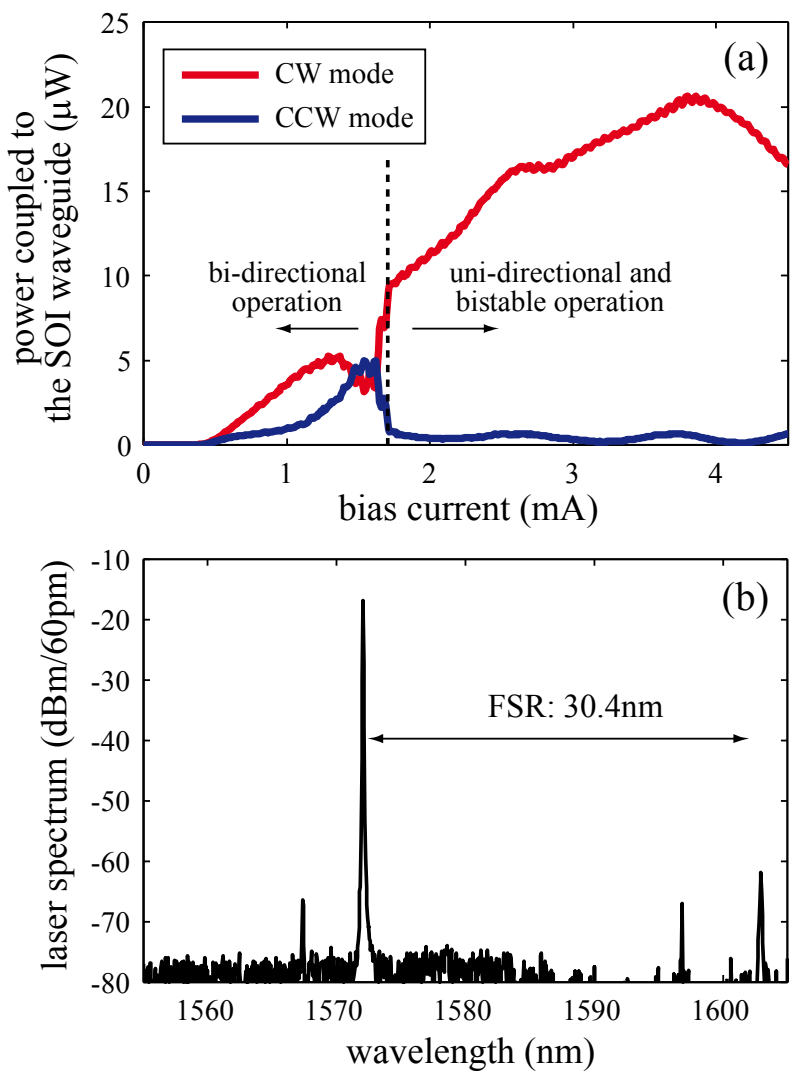

Figure 2 


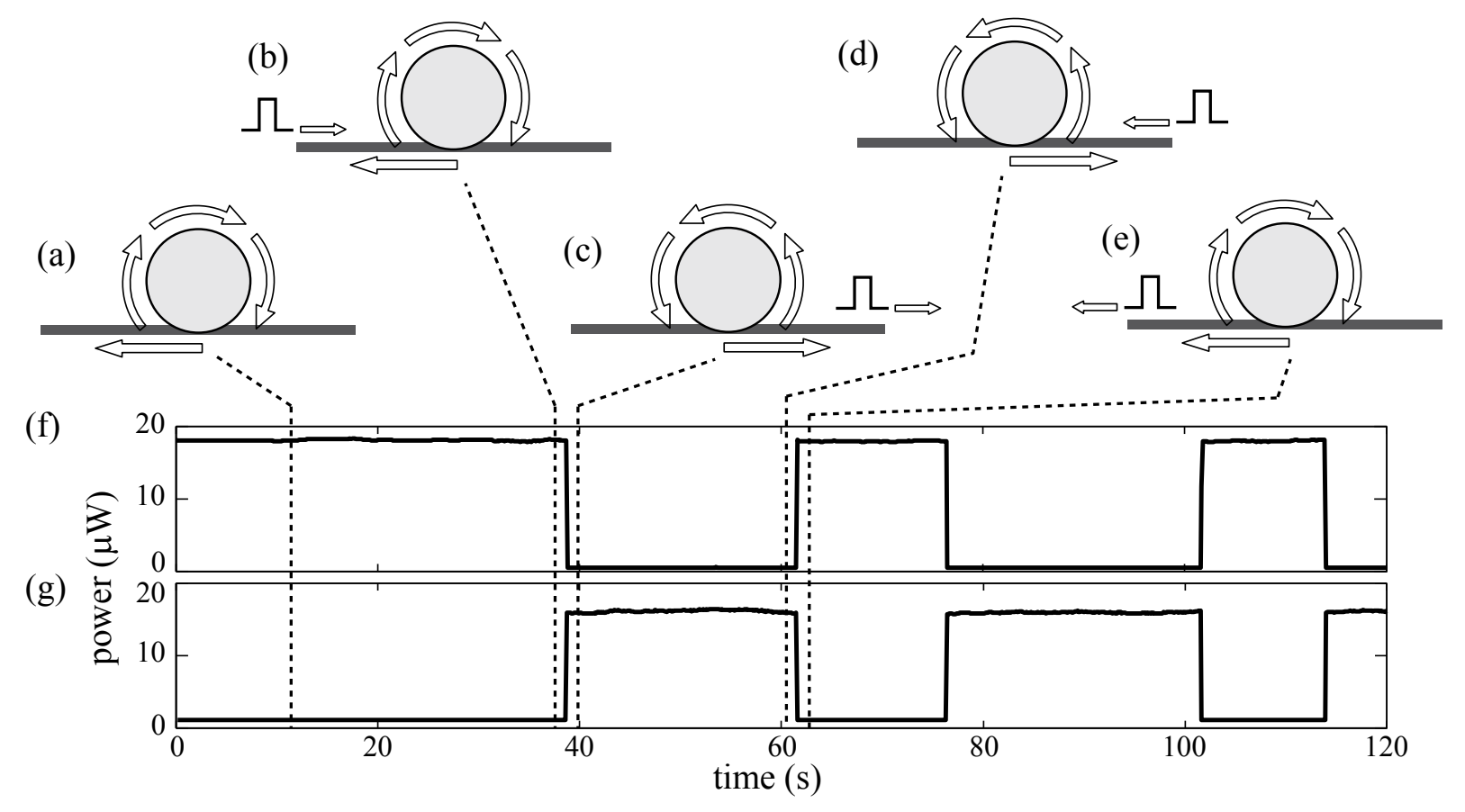

Figure 3 

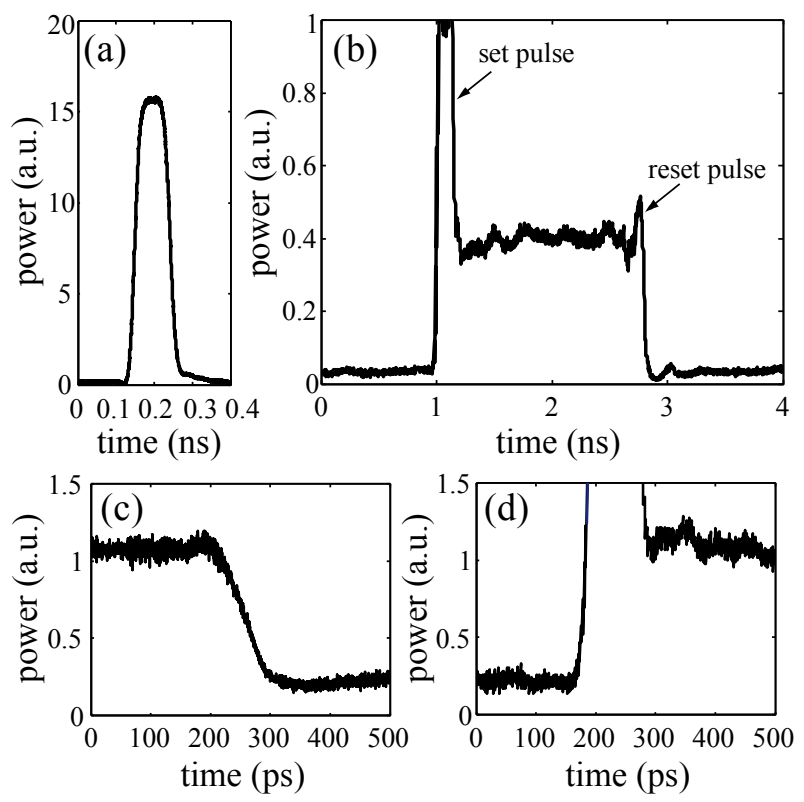

Figure 4 